\title{
Detecting Sponsored Recommendations
}

\author{
SUBHASHINI KRISHNASAMY, RAJAT SEN, and SANJAY SHAKKOTTAI, \\ University of Texas at Austin \\ SEWOONG OH, University of Illinois at Urbana-Champaign
}

\begin{abstract}
With the vast number of items, Web pages, and news from which to choose, online services and customers both benefit tremendously from personalized recommender systems. Such systems additionally provide great opportunities for targeted advertisements by displaying ads alongside genuine recommendations. We consider a biased recommendation system in which such ads are displayed without any tags (disguised as genuine recommendations), rendering them indistinguishable to a single user. We ask whether it is possible for a small subset of collaborating users to detect such bias. We propose an algorithm that can detect this type of bias through statistical analysis on the collaborating users' feedback. The algorithm requires only binary information indicating whether a user was satisfied with each of the recommended item or not. This makes the algorithm widely appealing to real-world issues such as identification of search engine bias and pharmaceutical lobbying. We prove that the proposed algorithm detects the bias with high probability for a broad class of recommendation systems when a sufficient number of users provides feedback on a sufficient number of recommendations. We provide extensive simulations with real datasets and practical recommender systems, which confirm the trade-offs in the theoretical guarantees.
\end{abstract}

Categories and Subject Descriptors: H.4 [Information Systems Applications]: Miscellaneous

General Terms: Algorithms

Additional Key Words and Phrases: Recommender systems, native advertising, sponsored content, anomaly detection, bias detection

ACM Reference Format:

Subhashini Krishnasamy, Rajat Sen, Sanjay Shakkottai, and Sewoong Oh. 2016. Detecting sponsored recommendations. ACM Trans. Model. Perform. Eval. Comput. Syst. 2, 1, Article 6 (November 2016), 29 pages. DOI: http://dx.doi.org/10.1145/2988543

\section{INTRODUCTION}

The growth of online services has provided a vast variety of choices to users. This choice exists today in multiple domains, including e-commerce with a variety of products and online entertainment (Netflix, Pandora). With users having to choose from an overwhelming set of items, recommender systems have become indispensable in easing the information overload and search complexity. Recommender systems are not restricted to retail businesses. A search engine like Google can be viewed as a recommendation engine that helps users find relevant information by ranking the search results according to their search criteria, history, and other personal information. Social networking

A shorter version of this work was accepted at ACM SIGMETRICS 2015 [Krishnasamy et al. 2015]. This work was supported by National Science Foundation grant CNS-1320175 and Army Research Office grant W911NF-14-1-0387.

Authors' addresses: S. Krishnasamy, R. Sen, and S. Shakkottai, Department of Electrical and Computer Engineering, The University of Texas at Austin, Austin, TX 78701; emails: subhashini.kb@utexas.edu, rajat. sen@utexas.edu, shakkott@austin.utexas.edu; S. Oh, Industrial and Enterprise Systems Engineering Department, University of Illinois at Urbana-Champaign, Urbana, IL 61801; email: swoh@illinois.edu.

Permission to make digital or hard copies of part or all of this work for personal or classroom use is granted without fee provided that copies are not made or distributed for profit or commercial advantage and that copies show this notice on the first page or initial screen of a display along with the full citation. Copyrights for components of this work owned by others than ACM must be honored. Abstracting with credit is permitted. To copy otherwise, to republish, to post on servers, to redistribute to lists, or to use any component of this work in other works requires prior specific permission and/or a fee. Permissions may be requested from Publications Dept., ACM, Inc., 2 Penn Plaza, Suite 701, New York, NY 10121-0701 USA, fax +1 (212) 869-0481, or permissions@acm.org.

(c) 2016 ACM 2376-3639/2016/11-ART6 $\$ 15.00$

DOI: http://dx.doi.org/10.1145/2988543 
sites like Twitter and Facebook display Tweets and news feeds based on users' past behavior and their connections to other users. News portals like Yahoo! News also present personalized content to online news readers.

Personalized recommender systems serve as an attractive platform for advertisers to reach their targeted consumers. It is now customary to see ads alongside other genuine recommendations in many of the Web sites that provide recommendation services. One can distinguish these ads from genuine recommendations, for example, by the location of their placement or their special tags. But recommendation engines are not legally obliged to facilitate such distinction and could possibly serve these ads mixed with genuine recommendations in a manner that renders them indistinguishable to users. Such advertising is commonly known in the online marketing world as native advertising.

According to the Interactive Advertising Bureau (IAB) [IAB 2013], native advertising aims "to deliver paid ads that are so cohesive with the page content, assimilated into the design, and consistent with the platform behavior that the viewer simply feels that they belong." Although native advertising is a relatively new phenomenon, it is expanding rapidly according to the numerous surveys conducted on sponsored content. According to a 2013 survey by the native advertising exchange Hexagram and the digital consultancy firm Spada [Hexagram 2014], 62\% of publishers offered native advertising at the time of the survey and another $16 \%$ planned to do so within a year. A survey by eMarketer [2014] revealed that marketing agencies see huge potential in native ads-almost three quarters of marketers already use native advertising and only $7 \%$ do not plan to use it in future. Spending on native advertising in the United States is predicted to increase $\$ 1.3$ billion in 2013 to $\$ 9.4$ billion in 2018 . This explosion is fueling the growth of native advertising as a business ecosystem with marketing agencies as consumers, publishers as media providers, and companies such as Hexagram, HubSpot, and Nudge offer tools to facilitate this exchange of digital media space. Indeed, several watchdog groups (both federal and industry sponsored) recognize this problem and have issued guidelines (e.g., the Federal Trade Commission (FTC) [FTC 1983] and the IAB [2010, 2013]).

A biased recommender system that shows paid ads and sponsored content without being transparent about them can have far-reaching consequences. These include user dissatisfaction with the recommendations [Beel et al. 2013]. A survey by Facebook showed that users find sponsored ads mixed with genuine posts in their news feed more annoying than the explicit, well-separated ads [Albergotti 2014]. Social and political consequences of bias in the context of media and online content have also been studied [Entman 2007; Introna and Nissenbaum 2000; Epstein 2014].

Thus, a basic question of interest to consumers is whether or not biased recommendations can be detected. It would be beneficial for watchdog agencies and groups that enforce compliance of ad disclosure policies [American Association of Advertising Agencies 2009; FTC 1983, 2013] to have suitable tools to detect sponsored recommendations that do not have explicit tags. Furthermore, it could also be used by businesses to monitor their own products or their competitor's products. As an example, India's antitrust watchdog, the Competition Commission of India (CCI), recently filed charges against Google for rigging its search results in favor of its own products and services [Gupta 2015]. The charges were based on an investigation of complaints from various competitors spanning businesses centered around search, social networks, and e-commerce. Automated tools that indicate potential biases in recommendations would be of utility to these type of agencies.

Modern recommender systems, in general, consist of two components: (i) learning individual preferences from user feedback and (ii) recommending items to users based on the estimated preferences. This combination of learning and recommending is bound to be noisy (the learning phase will explore individual preferences typically by 
presenting "random" recommendations), and several recommendations to users will likely be ineffective. Critically, both noise and bias manifest as bad recommendations to users. However, noise is benign and is a consequence of learning, whereas bias is systematic and is to be deprecated.

The problem of detecting bias in its most general sense is a broad topic and outside the scope of this article. Here we focus on a detecting a specific type of bias as described previously, where recommendation engines show sponsored content to users contrary to their preferences without being transparent about their recommendations. These biased systems systematically favor a few items over other better or at least equally good items, contrary to what an objective or unbiased system would do.

It should be noted that with most service providers being nontransparent about their recommendation strategies, one cannot hope to know the exact statistical profile of the recommendation engine a priori. Therefore, the key is to identify the primary features that can be used to differentiate between the two types without any a priori knowledge about the particulars of the recommendation strategy. For instance, one could consider the average rating or the average number of ineffective recommendations as the performance measure and make a decision based on a threshold parameter. However, as we also demonstrate through simulations, such a basic algorithm based solely on average performance cannot distinguish between deliberate systematic bias and innocuous random errors. This brings us to the following key question: can we develop a better method to expose a biased recommender system?

\subsection{Contributions of This Article}

We say that a recommendation engine is biased if it systematically favors a small set of items over other items in the database irrespective of user preferences. On the other hand, we say that a recommendation engine is objective if it satisfies a simple monotonic property in its recommendations to users-better suited items are given higher priority (in a statistical sense). The primary goal of this article then is to develop algorithms to answer the following question: can a meaningful distinction be drawn between objective and biased recommendation engines?

Binary feedback anomaly detector algorithm. We propose an anomaly detection algorithm that we call binary feedback anomaly detector (BiAD), which uses a statistical approach to identify a biased recommendation engine. Under appropriate conditions on the size of the ad pool, the aggressiveness of the biased recommender system, and the number of users/samples, we show that BiAD correctly (with high probability) distinguishes between objective and biased recommendation engines.

The algorithm leverages user collaboration and is based on the observation that a biased system is typically characterized by the occurrence of a large number of ineffective recommendations in a small set of items. On the contrary, giving higher priority to more effective items, as in an objective recommender system, precludes such concentration in a small set. Notably, since the users are not aware of the set of items, the $\mathrm{BiAD}$ algorithm is adaptive-as the recommender system learns users, the users "learn" the recommender system. Further, our algorithm relies only on binary feedback on the effectiveness of the recommendation. Although we assume in our model that this feedback is explicitly provided by the users, obtaining only implicit feedback may be feasible in some practical scenarios. In such settings, a binary feedback model is extremely useful since it is easy to interpret implicit feedback in terms of effectiveness of the recommendation. In Section 4.3, we give an example of a situation where only implicit feedback can be obtained and a binary model of effectiveness is applicable. Finally, the BiAD algorithm also works for a large class of recommender systems, as our model does not place any constraints on the recommendation engine other than 
mild statistical conditions. We finally present extensive simulation results that cover various types of recommender systems and datasets to illustrate the wide applicability of the algorithm.

\subsection{Related Work}

Following the recent successes of the targeted advertising services, several empirical studies have investigated the effects of displaying sponsored content alongside organic content [Beel et al. 2013; Ghose and Yang 2009; Tucker 2012]. It is empirically shown in Beel et al. [2013] that customers are less likely to select recommendations that are tagged as "advertisement" or "sponsored," motivating the advertisers to remove such tags. There have also been attempts to explain such effects through theoretical models [Yang and Ghose 2010; Bergemann and Bonatti 2011]. In addition, several researchers have worked on designing systems and algorithms from the content provider's perspective for revenue maximization through efficient auction of the ad space [Lahaie et al. 2007] and from the advertiser's perspective for effectively reaching the target audience [Rusmevichientong and Williamson 2006; Turpin and Katz 2008].

Our work, on the other hand, deals with identification of covert interaction between content providers and advertisers, specifically of promotional content being passed off as editorial content or recommendations. This problem falls under the broad class of problems called anomaly detection, which generally involve identification of unusual patterns in a system. Examples of such problems include network intrusion detection, fraud detection, and so on [Lazarevic et al. 2003; Bolton and Hand 2002]. Prior work on anomaly detection in recommender systems exists from the perspective of a recommendation engine as a victim of false user profile injections [Burke et al. 2006; Mobasher et al. 2007]. To the best of our knowledge, ours is the first work that considers the problem from the users' perspective and proposes a mechanism for detection of bias in recommendation engines.

There is vast literature in the broader context of anomaly detection-a comprehensive survey can be found in Chandola et al. [2009]. Various techniques of anomaly detection that include classification, clustering, statistical, information-theoretic methods, and so on are applicable depending on the type of input data, the type of anomaly, and the desired output. Clustering and neighborhood-based techniques have been proposed for many of the applications domains that seek to label a large collection of data points as either normal or anomalous [Ramaswamy et al. 2000; Papadimitriou et al. 2003; Yu et al. 2002; He et al. 2003; Labib and Vemuri 2002]. These techniques are based on differentiation of features through comparison of multiple data points against each other. Another category of techniques called classification [Schölkopf et al. 2001; Hawkins et al. 2002; Barbara et al. 2001; Song et al. 2002; Mahoney and Chan 2002] is based on using training data-data points that have been labeled a priori-to learn the appropriate features of anomalous behavior.

The preceding techniques, designed for multiple input data instances, cannot be applied to problems like the one considered in this article, which require detection of a single anomalous instance. For these problems, one has to rely on features specific to the problem to differentiate between normal and anomalous data instances. Our approach to the problem of detecting sponsored recommendations falls in the category of statistical techniques [Aggarwal and Yu 2001; Galeano et al. 2006; Agarwal 2007; Kruegel and Vigna 2003; Yeung and Chow 2002], following the classification of techniques in Chandola et al. [2009]. In this class of anomaly detection techniques, the features specific to the problem are characterized by a probabilistic model, and statistical inference tests are used to determine whether or not the input instance conforms to the representative model. 
In this setting, we make only mild assumptions in our model of the recommendation engine and do not restrict to a specific type of recommendation algorithm. This enables our algorithm to be applied to a large class of recommender systems.

\section{SYSTEM MODEL}

In this section, we describe our assumptions about the structural properties of objective and biased recommender systems by the means of a probabilistic model. This model does not include any particulars about the working of the recommendation engine and therefore typifies a broad class of recommender systems. Before we proceed to describe the model in detail, the salient features of this model are listed in the following:

-An objective recommendation engine has a fairly good estimate of user preferences.

-An objective recommendation engine follows the monotonic property-higher preference to higher-ranked items.

-A biased recommendation engine systematically gives preference to a small set of items irrespective of user tastes.

Notation. Our notation $O, \Omega, \Theta, o, \omega$ to describe the asymptotics of various parameters with increasing size of the database (total number of items in the database) is according to the standard Landau notation. We say that an event occurs with high probability if the probability of the event tends to 1 as the size of the database goes to infinity. We use $\mathbb{1}\{\cdot\}$ to represent the indicator function-that is,

$$
\mathbb{1}\{E\}:= \begin{cases}1 & \text { if event } E \text { occurs } \\ 0 & \text { otherwise. }\end{cases}
$$

Equality and inequality between random variables always refer to almost sure (with probability 1) conditions unless otherwise specified. For example, if $X$ and $Y$ are two random variables, then $X=Y$ implies that $X=Y$ almost surely. For any given matrix, $\mathbf{R}$, the $u^{\text {th }}$ row of $\mathbf{R}$ is represented by $\mathbf{R}_{u}$.

\subsection{User-Item Database}

The recommendation engine recommends products to users from a large database of $m$ items indexed from 1 to $m$. A user's opinion about an item is represented by a numerical value that we call the user's rating of that item. It should be noted that these ratings are only an implicit representation of true opinions of the users-the higher the rating, the better suited is the item for the user. We denote the user-item rating matrix for the entire database by $\mathbf{R}$, where rows indicate users and columns indicate items. We introduce a parameter called the efficacy threshold, denoted by $\eta$, which is used to represent opinions on a binary scale. We assume that a user is satisfied with a recommendation if the rating of the recommended item is greater than or equal to $\eta$. We refer to such a recommendation and item as being effective for that user.

Definition 2.1 (Effective and Ineffective). An item $i$ is effective for a user $u$ if the rating of that item by the user, $R_{u i}$ is at least $\eta$. Similarly, a recommendation is said to be effective for a user if the recommended item is effective. An item or recommendation that is not effective is said to be ineffective.

Let $f_{u}(\eta,[m])$ denote the number of items in the database $[m]$ whose rating is greater than or equal to $\eta$ for user $u$. In other words, it is the number of effective items in the database for user $u$.

Let us define the function $F: \mathbb{R} \times \mathbb{R}^{m} \rightarrow \mathbb{R}$ as follows:

$$
F\left(r, \mathbf{R}_{u}\right):=\left|\left\{i: R_{u i} \geq r\right\}\right|,
$$


where $R_{u i}$ is the $i^{t h}$ element of the $m$-length vector $\mathbf{R}_{u}$. This function is used to find the number of items whose rating exceeds value $r$ for any player $u$ if the ratings of all items in the database for player $u$ is given by $\mathbf{R}_{u}$. For example, if $\mathbf{R}_{u}$ is the row corresponding to player $u$ in the rating matrix, $\mathbf{R}$, then $F\left(\eta, \mathbf{R}_{u}\right)$ is equal to $f_{u}(\eta,[m])$, the number of effective items for user $u$. Similarly, $F\left(R_{u i}, \mathbf{R}_{u}\right)$ gives the rank of item $i$ for user $u$. Also note that for any given $\mathbf{R}_{u}, F\left(r, \mathbf{R}_{u}\right)$ is a nonincreasing function of $r$.

\subsection{Recommendation Engine}

We next describe the behavior of a recommendation engine using a probabilistic model. Let $\mathbb{1}_{u i}(t)$ indicate whether item $i$ has been recommended to user $u$ at time $t$ - that is,

$$
\mathbb{1}_{u i}(t):= \begin{cases}1 & \text { if item } i \text { is recommended to user } u \text { at time } t \\ 0 & \text { otherwise. }\end{cases}
$$

We make the following assumption about any recommender system: an item that has been recommended to a user once is not recommended to the same user again-that is, for any user $u$ and item $i, \sum_{t=1}^{\infty} \mathbb{1}_{u i}(t) \leq 1$.

2.2.1. Objective Recommendation Engine. An objective recommendation engine is considered to consist of two components: one is the learning strategy, which estimates the user-item rating matrix by the means of available feedback from users, and another is the recommendation strategy, which generates recommendations based on the estimated user preferences. Our model does not specify the details of the learning strategy except requiring that the output of the strategy (i.e., the estimate of the user-item matrix) be close to the original rating matrix, $\mathbf{R}$. Therefore, this model could be applied to a wide class of recommendation engines that estimate user preferences fairly well. Let the estimate of the rating matrix at time $t$ be denoted by $\hat{\mathbf{R}}(t)=\left[\hat{R}_{u i}(t)\right]$. This estimate is modeled as the sum of the original rating matrix and an additive noise matrix whose elements are independent across users, items, and time. This can be written as $\hat{\mathbf{R}}(t)=\mathbf{R}+\boldsymbol{\epsilon}(t)$, where $\boldsymbol{\epsilon}(t)=\left[\epsilon_{u i}(t)\right]$ is the noise matrix and $\epsilon_{u i}(t)$ is independent of all other random variables for all $u, u^{\prime}, i, i^{\prime}, t, t^{\prime}$.

The recommendation strategy uses the estimated user-item rating matrix $\hat{\mathbf{R}}(t)$ to make recommendations at time $t$. The following model characterizes the behavior of an objective recommendation strategy:

(1) Recommendations are made based on a user-item weight matrix, denoted by $\mathbf{W}(t)=$ $\left[W_{u i}(t)\right]$. This is a stochastic matrix (rows sum to one), which is updated based on the current estimate of the rating matrix, $\hat{\mathbf{R}}(t)$.

(2) Given the weight matrix, a user is given a recommendation by choosing an item randomly, independent of everything else, with weights given by the row corresponding to the user in the user-item weight matrix.

(3) At any time $t$, the weight matrix $\mathbf{W}(t)$ satisfies the following monotonic property: if $i$ and $j$ are two items that have not been shown to user $u$ and the ratings are such that $\hat{R}_{u i}(t) \geq \hat{R}_{u j}(t)$, then the weights satisfy $W_{u i}(t) \geq W_{u j}(t)$.

2.2.2. Biased Recommendation Engine. A biased recommendation engine marks a small set of items, $\mathcal{A}(\subseteq[\mathrm{m}])$ from the item database as ads. To make a recommendation to a user, with probability $\gamma$, independent of everything else, it chooses an item that has not been shown from the ad pool, $\mathcal{A}$. And with probability $1-\gamma$, it can follow any recommendation algorithm (e.g., an objective recommendation algorithm). We refer to $\gamma$ as the bias probability. Note that the strategy for showing ad items is unspecified except that no item is shown to a user twice. In particular, the engine may even customize its 
ad recommendations according to user tastes. As in the case of the complete database, let $f_{u}(\eta, \mathcal{A})$ denote the number of effective ads in the ad pool $\mathcal{A}$ for user $u$.

\subsection{Discussion of Assumptions}

Some of the assumptions in the preceding recommender system model are present only for ease of analysis. In the following, we discuss how they can be relaxed in practical settings:

(1) It is assumed that in any recommendation engine, an item once recommended to a user is not recommended to the same user again. This condition is required only to ensure that there are no repeated recommendations of sponsored advertisements that might be effective. Indeed, if all sponsored ad recommendations are effective, it would not be possible to distinguish them from genuine recommendations. This assumption can therefore be relaxed to require a sufficient number of ineffective ad recommendations in a biased recommender system. (A precise mathematical statement is given at the end of this discussion.)

(2) The noise in estimation of the user-item rating matrix is assumed to be additive independent and identically distributed noise. This can be replaced by a more general noise model in which the elements of the estimated user-item matrix are independent across users, items, and time. The independence assumption is used to model arbitrary errors that are unlikely to skew the estimated matrix in such a way as to give high preference to a small number of ineffective items uniformly across a large subset of users.

(3) We assume that a biased recommendation engine decides to show sponsored ads with probability $\gamma$ (bias probability) independent of everything else. This assumption, again, is used only for ease of exposition. It is sufficient to have $\Omega(\gamma)$ fraction of the total recommendations from the ad pool, not necessarily chosen at random.

To be mathematically precise, the assumptions discussed in points (1) and (3) can be relaxed to the following condition for the analytical results in this article to hold:

There exist constants $c_{1}, c_{2}>0$ such that for any $t_{1} \geq 0, t_{2}>t_{1}, n>0$, and any set of users $\mathcal{U}$ such that $|\mathcal{U}|=n$,

$$
\mathbb{P}\left[\sum_{l=t_{1}+1}^{t_{2}} \sum_{u \in \mathcal{U}, i \in \mathcal{A}} \mathbb{1}_{u i}(l) \geq c_{2} \gamma n\left(t_{2}-t_{1}\right)\right] \geq 1-e^{-c_{1} \gamma n} .
$$

In other words, for any set of $n$ users, if abiasedrecommendation engine makes $t$ recommendations to each user, then with probability at least $1-e^{-c_{1} \gamma n}$, the total number of recommendations from the ad pool among the nt recommendations is at least $c_{2} \gamma n t$.

\section{ANOMALY DETECTION ALGORITHM AND THEORETICAL RESULTS}

In this section, we describe the algorithm for detecting anomalous systems and provide analysis of Type I and Type II errors as in binary hypothesis testing.

\subsection{Anomaly Detection System}

The problem is to design a test to detect if a recommendation engine is biased. In other words, the test has to decide between the following two hypotheses:

$-H_{1}$ : "The recommendation engine is biased," and

$-H_{0}$ : "The recommendation engine is not biased."

It is similar to a hypothesis testing problem except the statistical distribution for the two hypotheses are not well defined. The only a priori knowledge that is assumed is 
the structure of a biased recommendation engine as specified in Section 2.2. But the specifics of various parameters in the recommendation engine such as bias probability $\gamma$ and the ad pool $\mathcal{A}$ are unknown. As in traditional hypothesis testing problems, we make use of multiple data points obtained from many users who constitute the anomaly detection system.

\subsection{Feedback Data}

The anomaly detection system consists of a set of $n$ players that is a subset of the user database in the recommendation system. Without loss of generality, we denote these players as users indexed from 1 to $n$ in the user database. These players give binary feedback (effective or ineffective) on the items recommended to them. The feedback can be subject to errors. We assume that the feedback given by user $u$ about any recommendation is in error with probability $\xi_{u}$ independent of everything else.

\subsection{Algorithm}

We now describe the BiAD algorithm [Krishnasamy et al. 2015], which uses the recommendations made to the players and their feedback to decide between one of the two hypotheses. In every round of recommendation, each player is recommended an item by the recommendation engine. In round $t$, the algorithm uses the feedback from the players and computes for each item the total number of players until that round who have been recommended that item and found the item ineffective. This number is denoted by $B_{i}(t)$ for item $i$. If the sum of the largest $\hat{A}(t)$ of these numbers among all the items is greater than or equal to a threshold $T(t)$, the recommendation engine is declared to be biased. Otherwise, the same procedure is repeated in the next round. If the algorithm does not declare the engine to be biased in $Q(m)$ rounds, then the hypothesis that the engine is biased is rejected. There are three parameters associated with the algorithm- $\hat{A}(t), T(t)$, and $Q(m)$-whose choice is governed by Equations (1) through (7). These parameters correspond to the following quantities:

$-\hat{A}(t)$ : This parameter corresponds to the size of the subset of movies used to compute the quantity of interest $S(t)$ in round $t$. This is elaborated on in the definition of $S(t)$ in Algorithm 1. The choice of this parameter that we use is given by Equation (1).

$-T(t)$ : This is a time-varying threshold in the algorithm that is compared to the quantity $S(t)$; the choice of this threshold is elaborated on in Theorem 3.1.

$-Q(m)$ : This is the maximum number of rounds of recommendation for which the algorithm operates before declaring a recommender engine as biased or objective.

The pseudocode for this algorithm is shown in Algorithm 1.

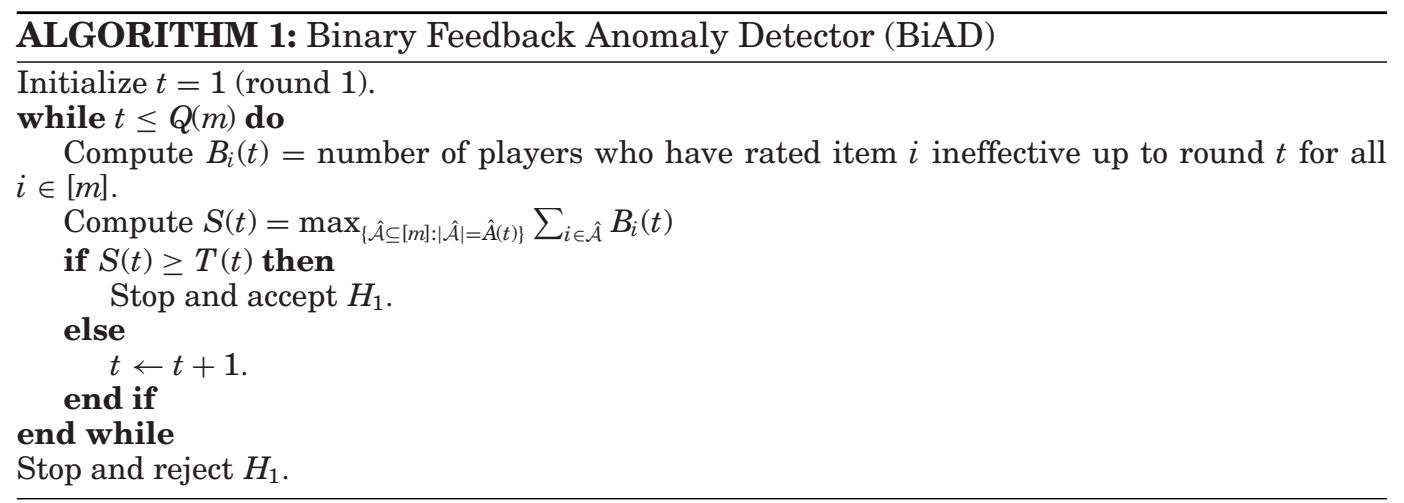


As opposed to the basic average test, this algorithm searches for a concentration of large number of ineffective items in a small set. Since the number of potential advertisements is unknown, this algorithm makes decisions in real time as it gets feedback from the players. The larger the size of the ad pool, the larger is the number of feedback samples required to detect a biased engine. (The trade-off between various parameters is discussed in detail in Section 4.) Therefore, the algorithm increases the size of the search set with progressing rounds of recommendation. In addition, note that the algorithm requires only binary feedback from the players-whether the recommendations are effective or ineffective-which explains the name of the algorithm.

The following theorem gives sufficient conditions for good performance of the algorithm. Unlike in general hypothesis-testing problems, we define Type I error, which corresponds to false positives, only for objective systems. On the other hand, Type II error is used to refer to missed detection in the case of a biased system. We do not give any guarantees for the class of recommendation engines that are neither objective nor biased.

THEOREm 3.1. Let the parameters in the detection algorithm, BiAD, satisfy the following equations:

$$
\begin{aligned}
\hat{A}(t) & =t, \\
& =\min \left\{n t, \exp \left(1+W\left(\frac{\hat{\beta}(t)}{e}\right)\right) \hat{p}(t)\right\},
\end{aligned}
$$

where $W(\cdot)^{1}$ represents the Lambert-W or product log function, and

$$
\begin{aligned}
\hat{\beta}(t) & =\left(\frac{(\hat{A}(t)+c) \log m}{\hat{p}(t)}-1\right) \\
\hat{p}(t) & =\exp \left(1+W\left(\frac{\beta(t)}{e}\right)\right) p(t), \\
\beta(t) & =\left(\frac{(\hat{A}(t)+c) \log m}{p(t)}-1\right)^{+}, \\
p(t) & =n t c^{\prime}+\max _{\{\hat{\mathcal{A}} \subseteq[m]:|\hat{\mathcal{A}}|=\hat{A}(t)\}}\left\{\sum_{u=1}^{n} \sum_{l=1}^{t} \mathbb{E}\left[P_{u}^{\mathcal{A}}(l)\right]\right\} \\
P_{u}^{\hat{\mathcal{A}}}(l) & =\sum_{i \in \hat{\mathcal{A}}} \frac{\mathbb{1}\left\{R_{u i}<\eta\right\}}{F\left(\hat{R}_{u i}(l), \hat{\mathbf{R}}_{u}(l)\right)-l+1},
\end{aligned}
$$

where $(\cdot)^{+}=\max (\cdot, 0)$ and $c, c^{\prime}$ are system design parameters.

$\overline{{ }^{1} \text { For any } z} \in \mathbb{R}, W(z) e^{W(z)}=z$. 
For $c=1 / 2$, BiAD gives the following guarantees on the error probabilities:

(I) Type I Error:

If the recommendation engine is objective, and if

(a) the feedback errors to the anomaly detection system are such that $\left\{\xi_{u}, u \in[n]\right\}$ satisfy $\frac{1}{n} \sum_{u=1}^{n} \xi_{u} \leq c^{\prime}$

(b) the estimation errors $\epsilon(t)$ of the recommendation engine, the number of rounds $Q(m)$ and $c^{\prime}$ are such that $\frac{p(t)}{n t} \leq \frac{1}{4} \forall 1 \leq t \leq Q(m)$, and

(c) the number of players $n=\omega(\log m)$,

then the probability that BiAD declares it to be anomalous is $O\left(\frac{Q(m)}{\sqrt{m}}\right)$.

(II) Type II Error:

If the recommendation engine is anomalous with an ad pool of size A, and if, along with Condition (I)a, the following conditions hold:

(a) the number of ads, $A \leq Q(m)$,

(b) the fraction of recommendations that are ads, such as the bias probability $\gamma \geq\left(\frac{e}{W(1)}\right)^{2} \frac{1}{(1-\delta)\left(1-c^{\prime}\right)} \max \left(\frac{\log m}{n}, \frac{p(A)}{n A}\right)$ for some constant $\delta \in(0,1)$, and

(c) $\sum_{u=1}^{n} f_{u}(\eta, \mathcal{A})=o(\gamma n A)$, where $f_{u}(\eta, \mathcal{A})$ is the number of effective ads for user $u$, then the probability that BiAD does not declare the system as anomalous within $A$ rounds is $e^{-\Omega\left(\left(1-c^{\prime}\right) \gamma n\right)}$.

The proof of this theorem is presented in Section 6 .

\section{DISCUSSION}

In this section, we discuss how the error probabilities depend on the parameters of the problem.

\subsection{Choice of Threshold}

Note that computation of the threshold function, $T(t)$, as specified in Theorem 3.1 (given by Equation (2)) requires knowledge of the noise statistics and also the players' opinions about all items in the database. More precisely, since $\hat{\mathbf{R}}(t)=\mathbf{R}+\boldsymbol{\epsilon}(t)$, computation of $\mathbb{E}\left[P_{u}^{\hat{\mathcal{A}}}(l)\right]$ (see Equation (7)) requires knowledge of $\mathbf{R}_{u}$ and also the distribution of estimation noise, $\epsilon_{u}(l)$. The noise statistics reflect the accuracy of the learning strategy of the recommendation engine, and it is possible that these statistics are unknown or cannot be estimated. Moreover, it might also be difficult to obtain the players' opinions about all items in the database. To overcome this difficulty, a practical implementation of the algorithm could use an approximation of the unknown quantity. We now propose one way to compute such an approximation. Note that

$$
\begin{aligned}
\mathbb{E}\left[P_{u}^{\hat{\mathcal{A}}}(l)\right] & =\mathbb{E}\left[\sum_{i \in \hat{\mathcal{A}}} \frac{\mathbb{1}\left\{R_{u i}<\eta\right\}}{F\left(\hat{R}_{u i}(l), \hat{\mathbf{R}}_{u}(l)\right)-l+1}\right] \\
& \leq \mathbb{E}\left[\sum_{i \in \hat{\mathcal{A}}} \frac{1}{F\left(\eta+\epsilon_{u i}(l), \mathbf{R}_{u}(l)+\boldsymbol{\epsilon}_{u}(l)\right)-l+1}\right],
\end{aligned}
$$

where the inequality follows since $F\left(r, \mathbf{R}_{u}(l)+\boldsymbol{\epsilon}_{u}(l)\right)$ is a nonincreasing function of $r$. We assume that the estimates of the ratings are not skewed in one direction, and therefore the noise has zero mean. Since the noise statistics are unknown, we could approximate the right-hand side of the preceding inequality by substituting the noise term with its mean. With this approximation, the right-hand side of the inequality can 
be substituted with

$$
\sum_{i \in \hat{\mathcal{A}}} \frac{1}{F\left(\eta, \mathbf{R}_{u}(l)\right)-l+1}=\frac{\hat{A}(t)}{f_{u}(\eta,[m])-l+1},
$$

where $f_{u}(\eta,[m])$ is the total number of effective items in the database for user $u$. Depending on the application, it might be relatively easy to estimate this number or at least estimate a lower bound for this number. As an example, one could roughly estimate that for every user there are $\sqrt{m}$ effective items among the $m$ items in the database. We observe in our simulations that a rough estimate of $f_{u}(\eta,[\mathrm{m}])$ is sufficient to obtain good results.

Note that overestimation (underestimation) of $T(t)$ decreases the probability of a Type I (Type II) error and increases the probability of a Type II (Type I) error. In other words, the higher the value of $T(t)$, the lower is the probability of a Type I error and the higher is the probability of a Type II error. Therefore, the risk associated with false positives and missed detection could serve as a guideline for the choice of the threshold function. In our simulations (Section 5), we propose a practical threshold function that gives a good balance between the two error probabilities for most scenarios.

\subsection{Effect of Parameters on Performance}

Theorem 3.1 gives guarantees on the asymptotic performance of $\mathrm{BiAD}$ as the size of the database grows large. These guarantees depend on various parameters in the algorithm as well as the recommendation engine. From the analytic bounds derived in Theorem 3.1, in this section we analyze the trade-off between these parameters to understand the conditions under which the algorithm shows good performance. We see that the theoretical results support our intuitive understanding about the conditions under which a biased system can be distinguished from an objective system. These results are also corroborated by our simulation results described in Section 5.

In Section 4.1, we consider the effect of the choice of the threshold parameter on the error probabilities. We now discuss the effect of other parameters.

Number of rounds in the test and size of the ad pool. It is seen (from Result I) that the upper bound on the probability of a Type I error increases with increasing number of rounds. This is expected, as it gives more chances to falsely declare a system biased. For a Type I error to go to zero as the size of the database goes to infinity, it is sufficient if $Q(m)=o(\sqrt{m})$.

Guarantees for detection of a biased engine (Result II) are dependent on various parameters. One of the conditions is that the ad pool is not very large (Condition (II)a). Specifically, it is sufficient if the size of the ad pool, $A$, is at most the maximum number of rounds of recommendations, $Q(m)$. Therefore, increasing $Q(m)$ (the number of rounds of testing) enables detection of larger ad pools but also increases the probability of a Type I error. Intuitively, a small ad pool conforms with our definition of a biased recommendation engine as one that favors a few items over many others and therefore facilitates easier detection.

Number of effective ads. For correct detection of a biased engine, it is also required that the average number of effective ads (averaged over all players) is not very large (Condition (II)c). A large number of effective ads enables the recommendation system to customize ads according to user tastes and is contradictory to our interpretation of a biased system that recommends ads that do not match with user preferences.

Number of players. The dependence on the number of players $n$ is seen in two respects: it determines the minimum bias probability at which detection is guaranteed 
(Condition (II)b) and also the probability of a Type II error. Both of these results show that a large number of players improves the prospect of correct identification, which can explained by the fact that a large sample size supports better statistical analysis.

Number of effective items. The minimum bias probability at which detection is ensured is also determined by the average number of effective items in the entire database. This can be seen from the term $\frac{p(A)}{n A}$ in Condition (II)b. The no estimation noise case $\left(\epsilon_{u i}(t)=0\right.$ for all $\left.u, i, t\right)$ is useful in understanding the term $\frac{p(A)}{n A}$. When there is no noise, $\frac{p(A)}{n A}=O\left(\frac{1}{n A} \sum_{u=1}^{n} \sum_{l=1}^{A} \frac{A}{f_{u}(\eta,[m])-l+1}\right)$. Therefore, $\frac{p(A)}{n A}$ has an inverse relation with the number of effective items in the database. This conveys that a large number of effective items facilitates better detection of a biased engine. Intuitively, a large number of effective items in the database helps in clearer demarcation of an objective engine from a biased one. With many effective items in the database, an objective system would have a higher probability of recommending effective items, whereas a biased system always makes at least $\gamma$ fraction of its recommendations from the ad pool where the number of effective ads is limited.

Bias probability. The more a biased engine recommends from the ad pool, the more apparent is its biased behavior. The fraction of total recommendations that are from the ad pool is captured by the bias probability, $\gamma$. We see that the probability of a Type II error decays exponentially with increasing $\gamma$, and also that larger $\gamma$ facilitates easier anomaly detection (Conditions (II)b, (II)c).

Choice of $c, c^{\prime}$. In the course of the proof of Theorem 3.1, we prove that for any choice of $c$, the Type I error is bounded by $O\left(Q(m) m^{-c}\right)$. Hence, by changing $c$ in the algorithm, one can control the error probability. However, the downside of increasing $c$ is that it effectively increases the threshold $T(t)$, which results in requiring the bias probability $\gamma=\Omega(\log m(1+(c / A)) / n)$.

Likewise, the choice of $c^{\prime}$ determines the trade-off between the algorithm's tolerance for errors in feedback and its ability to correctly identify a biased engine. This trade-off can be observed from the conditions given in Theorem 3.1. The higher the value of $c^{\prime}$, the greater is the range of feedback errors for which the guarantees hold (Condition (I)a). However, this results in poorer guarantees for detection of bias reflected by Conditions (I)b, (II)b, and higher Type II error probability.

\subsection{Applications}

The proposed anomaly detection algorithm is readily applicable in the retail market. It can identify recommender systems that dole out sponsored advertisements in the garb of personalized recommendations. In this era of personalization, there are numerous other applications, two of which are described in the following. These two examples also illustrate the advantage of $\mathrm{BiAD}$ in requiring simple binary feedback, allowing it to be applied in a wide variety of scenarios.

Search engine bias. Search engine bias is one of the most important ethical issues surrounding search engines, and its social implications have been studied for more than a decade [Introna and Nissenbaum 2000; Zimmer 2006; Epstein 2014]. There has been increasing awareness about the influence of search results ranking on election results in democracies. In Epstein [2014], experiments performed with 1,800 undecided voters in India revealed that alteration of search results can create a shift of $12.5 \%$ in voting decisions. The Stanford Encyclopedia of Philosophy [Tavani 2014] describes search engine bias as nonneutrality of search engines, where "search algorithms do not use objective criteria" or "favor some values/sites over others in generating their list of results for search queries." A sponsored search engine in the late 1990s called GoTo 
ranked its search results purely based on bids from advertisers [Elgesem 2008]. It was evidently unsuccessful due to users' mistrust of paid searches and was eventually acquired by Google. Google also uses an auction to sell ads but displays them physically separated from organic search results.

The pros and cons of enforcing transparency in the algorithms used for generating search results have been examined in Elgesem [2008] and Granka [2010]. Even in the absence of total transparency, anomaly detection systems such as BiAD could be useful in identifying bias in search engines. With personalization being extended to search results [Dou et al. 2007; Horling and Kulick 2009; Crook and Ahari 2011], search engines virtually act as recommendation engines. With a large number of potential search results, our model of recommender systems with a large database fits well in this problem where biased search engines correspond to biased recommender systems. To be more precise, personalized search result ranking algorithms like PageRank [Horling and Kulick 2009] can be viewed as the learning strategy of the recommendation system, whereas alteration of search results due to malicious reasons can be modeled as bias in the recommendation engine. Feedback from users can be obtained through their clicks on recommended links that implicitly indicate whether or not the search results were effective. In addition to search engines, this example can be extended to identify hidden sponsored advertisements in social networking sites and online news portals, all of which use personalization algorithms.

Pharmaceutical lobby. Pharmaceutical lobbying is another controversial issue that affects many parts of the world [Abraham 2002; Landers and Sehgal 2004; Lancet Editorial 2005; Shah 2010]. Among its many aspects, we focus on the marketing practices of large pharmaceutical companies that manipulate the opinions of doctors, health care providers, and lawmakers by providing biased information and through other tactics [Blumenthal 2004; Fugh-Berman and Ahari 2007]. There have been allegations that big drug companies influence physicians to prescribe their highly priced branded drugs even when other better or cheaper alternatives are available [Landefeld and Steinman 2009; Goldacre 2014].

Again, our interpretation of a biased recommendation engine is well suited to model this scenario. Since drugs are prescribed on a person-to-person basis, health care providers can be viewed as recommendation service providers who recommend drugs to patients, and the lobbying drug companies act as advertisers. A health care system that favors a few incompetent (expensive or ineffective) drugs despite the availability of other better (cheaper or more effective) alternatives matches well with our definition of a biased recommendation engine. With data samples consisting of prescriptions and their efficacy on patients, anomaly detection algorithms like BiAD could help watchdog agencies in identifying such malpractices.

\section{NUMERICAL RESULTS}

We evaluate our algorithm through offline simulations, with careful considerations for ensuring proximity to real-world scenarios.

\subsection{Simulation Setup}

In the following, we present a detailed description of the methods that we adopt in our simulations to replicate the different components of a recommender system.

User-item database. Estimating users' opinions about all items in a database is essential for real-world recommender systems, and hence for simulating those recommender systems as well. However, the ground truth on such a dataset is not available, because in existing datasets, each user typically only rates a small subset of items, and those ratings are also noisy and possibly biased. 
For a complete user-item rating matrix, we take available sparse datasets ([McAuley and Leskovec 2013; Bennett and Lanning 2007], MovieLens) and renormalize the ratings on a linear scale from 0 to 10 . The missing entries in the sparse matrix are then filled in using the matrix completion algorithm from Keshavan et al. [2010]. For the purpose of simulating real-world user opinions, we consider this completed matrix as ground truth. We evaluate our algorithm on three datasets:

D1: a subset of the Amazon cell phones and accessories dataset [McAuley and Leskovec 2013] with 3,671 users and 8,728 items,

D2: a subset of the Netflix Prize dataset [Bennett and Lanning 2007] with 2.951 users and 9.259 movies, and

D3: a subset of the MovieLens 10M ratings dataset with 3,671 users and 8,729 movies.

Recommendation engine. Due to nontransparency of recommendation strategies, it is not exactly known how recommendation engines behave. As a representation of the learning strategies used by these systems, we use two learning algorithms popular in the literature:

L1: matrix factorization (specifically, we use the inexact ALM method proposed in Lin et al. [2010]) and

L2: user-based collaborative filtering (with Pearson correlation as the similarity metric [Resnick et al. 1994]).

To simulate the temporal dynamics of a recommender system, the recommendation engine is initially supplied a sparse subset of the user-item ratings chosen according to a power-law degree distribution observed in real-world datasets [Huang et al. 2007]. (Specifically, the number of feedback entries from each user is chosen from a pareto $(3,3)$ distribution.) In each round of recommendation, the engine recommends one item to each user and observes users' feedback about the recommended items. It periodically updates its estimate of the users' preferences based on this feedback. In our experiments, we set the frequency of these updates to once in every five rounds.

It is natural for a recommendation engine to have an explore component to address the cold start problem and have wider coverage of the database [Li et al. 2010]. Therefore, in all of our experiments, we invoke random explore for 0.1 fraction of the recommendations made. In a recommendation meant for exploration, an item is chosen uniformly at random from the database, provided that it has not been shown previously.

For all other recommendations that do not explore, we use the following recommendation strategy. For each user, the items are ranked according to the estimated preferences. To make a recommendation, an objective recommendation engine chooses the highest ranked item among the items not yet recommended. The recommendation strategy of a biased engine follows the description in Section 2.2.2, in which for any recommendation, with probability $1-\gamma$, like an objective engine, it recommends the highest-ranked item, and with probability $\gamma$, it recommends an item from the ad pool. We consider two kinds of ad selection strategies: from the among the ads that have not already been recommended to the user,

A1: an ad item is chosen uniformly at random;

A2: the ad item that has the highest ranking is chosen.

Strategy A2 corresponds to customization of ads according to user tastes. Note that this is harder to detect than strategy A1, as it has higher likelihood of recommending effective ads.

Anomaly detection system. For players in the anomaly detection system, we randomly choose a subset of users from the dataset. In experiments that test the performance 
of the algorithm with increasing numbers of players, we choose the subset of players incrementally.

As explained in Sections 2 and 3, the algorithm requires feedback samples of efficacy of the recommendations made to the players. For our experiments, we adopt the characterization of efficacy used in our theoretical model given by Definition 2.1. Note that the number of effective items in the database for any user depends on the efficacy threshold $\eta$. To be able to test the algorithm for a different number of effective items, we choose a different efficacy threshold for each of the datasets. Specifically, we set $\eta=5.5,8.0$, and 8.8 for datasets D1, D2, and D3, which correspond to an average number of effective items of 80,250 , and 150 , respectively.

\subsection{Results}

We evaluate the performance of $\mathrm{BiAD}$ with variations in different parameters of the recommender system and the anomaly detection system. To demonstrate its effectiveness in different settings, we present performance results for various combinations of datasets (D1 through D3) and recommendation algorithms (L1 and L2, A1 and A2). An objective recommendation engine is represented by its learning algorithm (L1 or L2), whereas a biased recommendation engine is represented by its learning algorithm (L1 or L2) and its ad recommendation strategy (A1 or A2). Although we present experimental results for specific combinations for space limitations, other settings give similar trade-offs. Specifically, the simulation results corroborate our theoretical analysis of the trade-offs between various parameters in Section 4.

We now describe how the performance depends on the choice of various parameters in BiAD. We set the parameter $\hat{A}(t)$ according to Equation (1) in all simulations. Other parameters of the algorithm are discussed in the following.

Threshold. As explained in Section 4.1, varying the threshold parameter $T(t)$ in the algorithm affects Type I and Type II error probabilities in opposite ways. Using lower values of threshold increases the probability of a Type I error and decreases that of a Type II error. The threshold given by Equation (2) is designed to ensure, irrespective of the number of players in the anomaly detection system, low probability of a false positive (Type I error) even when the estimated user preferences are noisy. This is especially important if the risk associated with false implication of an objective recommendation engine is high.

We observe that a less conservative threshold gives a better balance between the two types of errors. Specifically, we use a threshold that can be proved to guarantee low error rates under the assumption that the recommendation engine's estimation of user preferences are accurate. This threshold, denoted by $T^{\prime}(t)$, is equal to the value of $\hat{p}(t)$ given by Equation (4). In all of our simulations, we show the performance of BiAD for both of these threshold choices. Simulation results show that $T^{\prime}(t)$ gives better performance than $T(t)$ except in one case (Figure 2(b)), where those two choices give similar performance.

In both of these thresholds, $\mathbb{E}\left[P_{u}^{\hat{\mathcal{A}}}(l)\right]$ in Equation (6) is substituted with the righthand side of (8). This requires knowledge of the number of effective items for each player in the anomaly detection system. In our simulations, BiAD approximates this with the average number of effective items for all users. Effectively, it uses the following value of $p(t)$ instead of Equation (6):

$$
p(t)=\sum_{l=1}^{t} \frac{n \hat{A}(t)}{\tilde{f}([m])-l+1}
$$




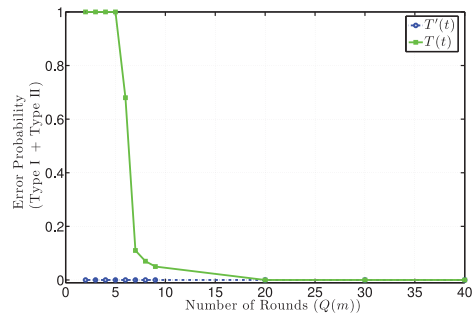

(a) Dataset: D1, Algorithm: L2 + A1, $n=100, \gamma=0.45, A=8$.

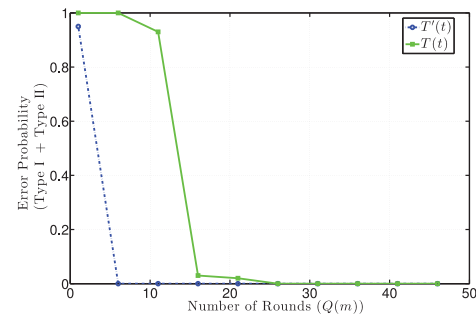

(b) Dataset: D2, Algorithm: L2 + A2, $n=100, \gamma=0.35, A=8$.

Fig. 1. Number of rounds in the test. $Q(m)$ affects the number of ads that can be detected-at least 8 and 15 rounds are required for $T^{\prime}(t)$ and $T(t)$, respectively.

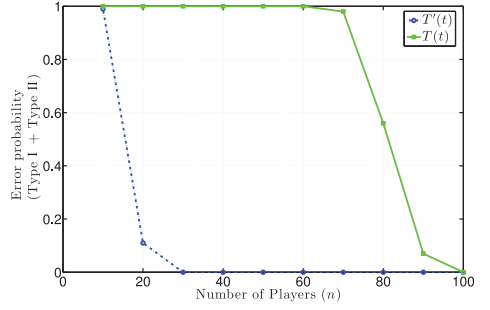

(a) Dataset: D1, Algorithm: L1 + A1, $A=$ $8, \gamma=0.45$.

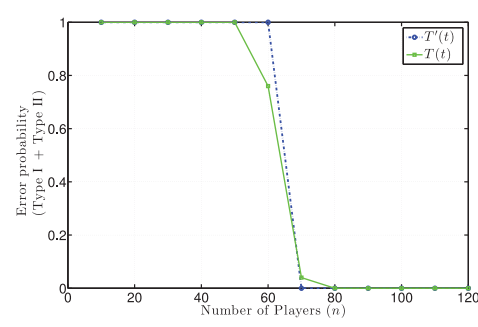

(b) Dataset: D2, Algorithm: L1 + A1, $A=8, \gamma=0.35$

Fig. 2. Performance improves with the number of collaborating users $n$.

where $\tilde{f}([\mathrm{~m}])$ is an estimate of the average number of effective items in the database $[\mathrm{m}]$. We assume that this average number is not very difficult to estimate, and in all of the results, unless specified, BiAD has an accurate estimate of this number.

Number of rounds in the test. The number of rounds of recommendation $Q(m)$ affects the error probability. This is seen in Figure 1, which shows the variation of sum of Type I and Type II errors with $Q(m)$. The Type I error rate is in fact close to zero for all values of $Q(m)$ for both the thresholds, so the plots effectively show Type II error rates. Theorem 3.1 guarantees detection of a biased engine if $Q(m) \geq A$. The plots show that BiAD detects eight ads if $Q(m)$ is at least 8 and 15 for $T^{\prime}(t)$ and $T(t)$, respectively. For all remaining simulations, we set the parameter $Q(m)=40$.

Number of players. A larger number of players in the anomaly detection system indicates a higher number of input samples to the algorithm, and as expected, the algorithm performs better as this number increases. In Figure 2, we plot the sum of Type I and Type II error rates with increasing numbers of users. To detect a biased engine with the specified value of $\gamma$, these plots show that 70 and 100 players, respectively, are sufficient when $T^{\prime}(t)$ and $T(t)$ are chosen to be the threshold parameter. We use 100 players in all other simulations.

In addition to the choice of parameters in the algorithm, various aspects of the recommender system affect the performance of BiAD. These are described in the following.

Size of the database. Theorem 3.1 shows that BiAD performs well for recommender systems with large item databases. Databases of varying size are constructed by subsampling items from the original dataset. Figure 3 shows the variation of Type I and Type II errors with the size of the database. $T(t)$ and $T^{\prime}(t)$ have very similar performance for the parameters in this experiment. The plot shows that for detection of eight 


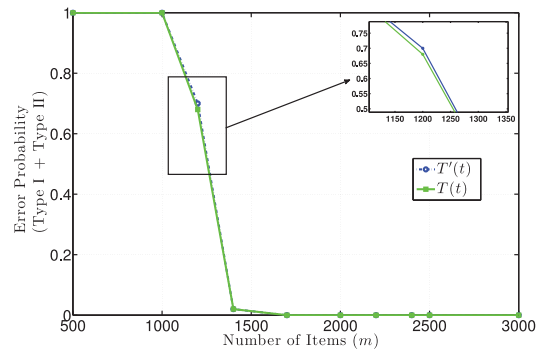

Fig. 3. Variation of Type I and Type II error rates with the size of the dataset. Dataset: D2, Algorithm: $\mathrm{L} 1+\mathrm{A} 2, A=8, \gamma=0.35, n=100$. When there are more choices to recommend, the user satisfaction with objective recommender systems improves, making detection easier.

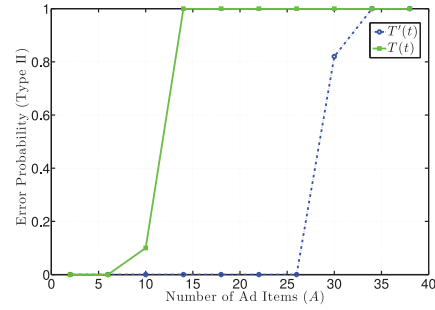

(a) Dataset: D1, Algorithm: L1 + A1, $n=100, \gamma=0.45$.

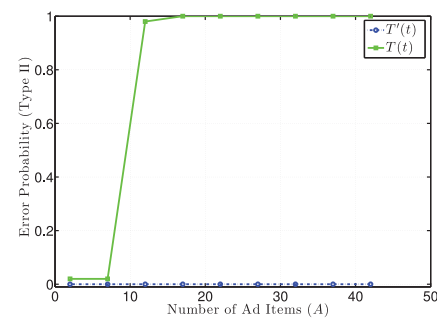

(b) Dataset: D2, Algorithm: L2 + A2, $n=100, \gamma=0.35$.

Fig. 4. As the size of the ad pool $A$ increases, the (personalized) ads become similar to effective recommendations, making it hard to detect (Type II error is large).

ads recommended $35 \%$ of the time, the algorithm is effective for databases having more than 1,500 items.

We now demonstrate that $\mathrm{BiAD}$ has been appropriately designed to identify biased engines that systematically recommend (make a sizable fraction of recommendations) from a small ad pool.

Size of the ad pool. Theorem 3.1 shows that BiAD guarantees detection of a biased engine that has a small ad pool. This same effect is also observed in simulations. Figure 4 shows the rate of missed detection (Type II error rate) with varying sizes of the ad pool. It is seen that both thresholds perform well for small numbers of ads, whereas threshold $T^{\prime}(t)$ can detect an ad pool smaller than 25 items.

Bias probability. The bias probability $\gamma$ quantifies the intensity of bias of the recommendation engine. Plots (Figure 5) for a Type II error rate with $\gamma$ show that more biased (higher $\gamma$ ) engines are easier to detect.

Average feedback error $\left(c^{\prime}\right)$. Theorem 3.1 shows that the probability of detecting a biased recommender system goes down with an increase in feedback error $c^{\prime}$. This can be observed in Figure 6, where a Type II error is plotted against increasing $c^{\prime}$ while all other parameters are held constant. In Figure 7, a Type II error is plotted against bias probability $(\gamma)$ in the presence of a feedback error of $10 \%$. With 1,000 players, the threshold $T^{\prime}(t)$ has zero error in the presence of feedback error for a bias probability as low as 0.2 .

With increasing feedback error, higher numbers of players are required to obtain performance comparable to the setting when there is no feedback error. Therefore, in practice, the effect of feedback errors can be mitigated by having sufficient numbers 


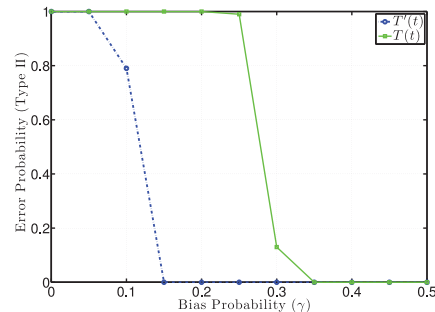

(a) Dataset: D2, Algorithm: L1 + A2, $n=100, A=8$.

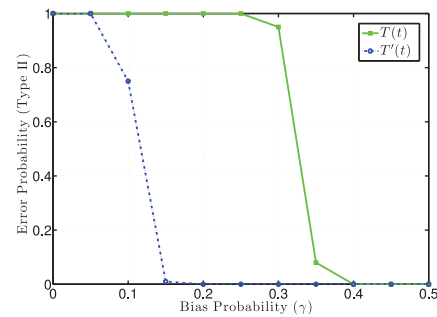

(b) Dataset: D3, Algorithm: L1 + A2, $n=100, A=8$.

Fig. 5. Type II error rate decreases as bias probability $\gamma$ increases.

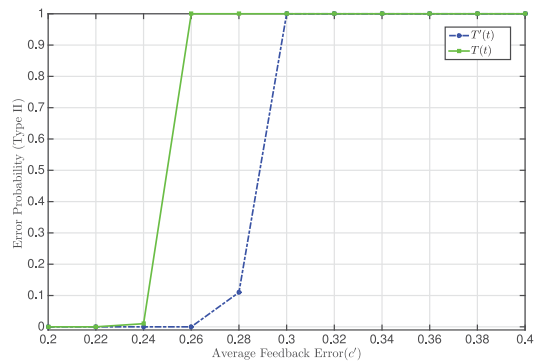

Fig. 6. Variation of Type II error rates with feedback error probability $\left(c^{\prime}\right)$. Dataset: D2, Algorithm: L1 + A1, $A=8, \gamma=0.5, n=2,000, Q(m)=10$. Error in detection increases with the increase in feedback error.

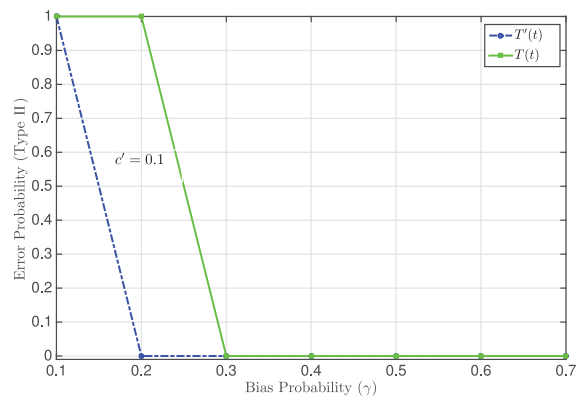

Fig. 7. Variation of Type II error versus bias probability $(\gamma)$ in the presence of feedback error. Dataset: D2, Algorithm: L1 + A1, $A=8, n=1,000, Q(m)=10$. Performance of detection strategy decreases with an increase in feedback error rate.

of participating players in the system. Note that for binary feedback, an error implies a complete flip in feedback, and therefore even a small percentage of errors can be significant. The preceding simulations demonstrate the robustness of BiAD against feedback errors.

Estimate of the number of effective items. In all of the preceding simulations, it is assumed that $\mathrm{BiAD}$ has an accurate estimate of the average number of effective items $(\tilde{f}([m]))$, which is used to determine the threshold parameter in the algorithm (see Equation (9)). Note that overestimation of this parameter lowers the threshold parameter, thereby increasing the probability of Type I error and decreasing the probability of Type II error. Figure 8 shows the effect of variations in this estimate for dataset D3, which has an average of 150 effective items. We observe that $T^{\prime}(t)$ performs well for 


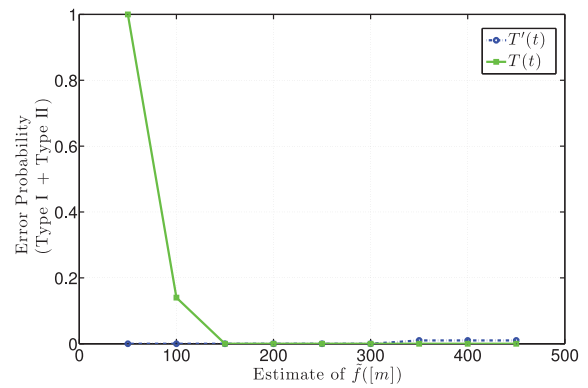

Fig. 8. Variation of Type I and Type II error rates with perturbations in the algorithm's estimate of the average number of effective items $\tilde{f}([m])$. Dataset: D3, Algorithm: L1 + A1, $A=8, \gamma=0.4, n=100$.

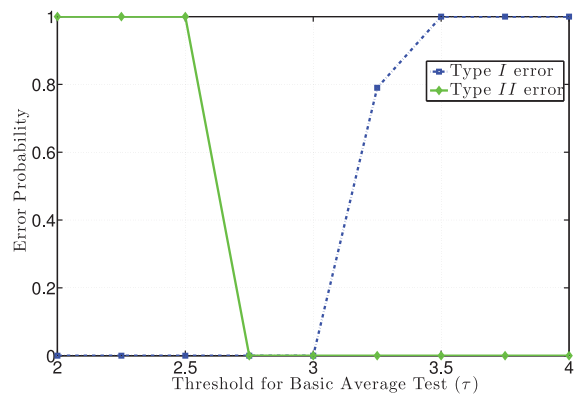

Fig. 9. Variation of Type I and Type II error rates with threshold $\tau$ for the basic average test shows that the naive approach is sensitive to the choice of parameter $\tau$. Dataset: D1, Algorithm: L1 + A1, $A=8, \gamma=0.45, n=100$, Explore Probability $=0.1$.

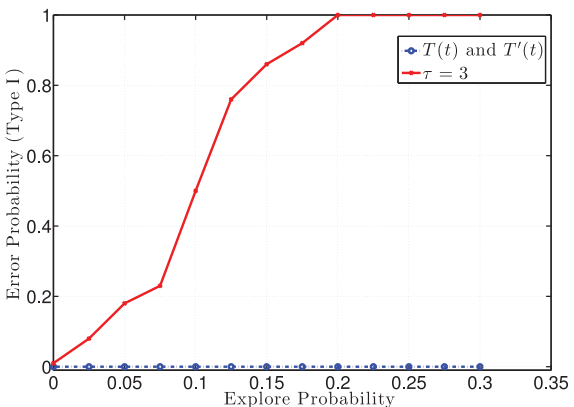

Fig. 10. Variation of Type I error rates with variation in explore probability shows that the threshold for the basic average test is sensitive to the value of explore probability. Dataset: D1, Algorithm: L1, $n=100$.

a wide range of estimates. In the case of $T(t)$, it is safer to overestimate the parameter $\tilde{f}([m])$ than to underestimate it.

\subsection{Ineffectiveness of Basic Average Test}

As explained in Section 1, we demonstrate the inability of the basic average test to distinguish between random errors and deliberate promotion of ads. This test computes the average rating across all recommendations and decides between the two hypotheses based on a threshold parameter. With the specifics of the recommendation strategy (explore probability) unknown, it is difficult to estimate the right threshold value. For an explore probability of 0.1 , Figure 9 shows the performance of the basic average test for different values of the threshold, denoted as $\tau$. It is seen that threshold values around 3 give the best performance. However, as shown in Figure 10, this same threshold value fails for other values of explore probability. For example, the basic average test falsely declares an objective recommendation engine with $20 \%$ explore probability as biased. This shows that the correct choice of $\tau$ is sensitive to the explore probability. In contrast, note that BiAD has nearly a zero Type I error rate for all values of explore probabilities.

\section{PROOF OF THEOREM 3.1}

Before we provide the proof of the main theorem, we state and prove two lemmas used in proving the main theorem. 
Lemma 6.1. For any objective recommendation algorithm and for any user $u$, item $i$, and time $t<\left(F\left(\hat{R}_{u i}(t), \hat{\mathbf{R}}_{u}(t)\right)+1\right)$, the probability that item $i$ is recommended to user $u$ at time $t$ is upper bounded by

$$
W_{u i}(t) \leq \frac{1}{F\left(\hat{R}_{u i}(t), \hat{\mathbf{R}}_{u}(t)\right)-t+1} .
$$

Proof.

$$
\begin{aligned}
1 & \stackrel{(a)}{=} \sum_{j=1}^{m} W_{u j}(t) \\
& \geq \sum_{j=1}^{m} \mathbb{1}\left\{\hat{R}_{u j}(t) \geq \hat{R}_{u i}(t)\right\}\left(1-\sum_{l=1}^{t-1} \mathbb{1}_{u i}(l)\right) W_{u j}(t) \\
& \stackrel{(b)}{\geq} \sum_{j=1}^{m} \mathbb{1}\left\{\hat{R}_{u j}(t) \geq \hat{R}_{u i}(t)\right\}\left(1-\sum_{l=1}^{t-1} \mathbb{1}_{u i}(l)\right) W_{u i}(t) \\
& =\left|\left\{j: \hat{R}_{u j}(t) \geq \hat{R}_{u i}(t), \sum_{l=1}^{t-1} \mathbb{1}_{u j}(l)=0\right\}\right| W_{u i}(t) \\
& \left.\geq||\left\{j: \hat{R}_{u j}(t) \geq \hat{R}_{u i}(t)\right\}|-|\left\{j: \sum_{l=1}^{t-1} \mathbb{1}_{u j}(l) \neq 0\right\} \mid\right) W_{u i}(t) \\
& =\left(F\left(\hat{R}_{u i}(t), \hat{\mathbf{R}}_{u}(t)\right)-(t-1)\right) W_{u i}(t),
\end{aligned}
$$

where $(a)$ and (b) follow from the characterization of an objective recommendation algorithm in Section 2.2.1-equality $(a)$ due to the fact that $\mathbf{W}(t)$ is a stochastic matrix (Property 1) and inequality $(b)$ due to the monotonic property satisfied by the weight matrix (Property 3). The preceding inequality gives the desired bound in (10) for $t<$ $\left(F\left(\hat{R}_{u i}(t), \hat{\mathbf{R}}_{u}(t)\right)+1\right)$.

Lemma 6.2. Let $\left\{X_{i}, i=1, \ldots, k\right\}$ be independent random variables with range $[0,1]$ and mean $\left\{p_{i}, i=1, \ldots, k\right\}$, and let $\sum_{i=1}^{k} p_{i} \leq p<k$. Then,

$$
\mathbb{P}\left[\sum_{i=1}^{k} X_{i} \geq T\right] \leq \exp \left(-T \log \left(\frac{T}{p}\right)+T-p\right) \forall k>T>p .
$$

Using the Chernoff bound for independent random variables, we have, for any $\theta>0$,

$$
\begin{aligned}
\mathbb{P}\left[\sum_{i=1}^{k} X_{i} \geq T\right] & \leq e^{-\theta T} \prod_{i=1}^{k} \mathbb{E}\left[e^{\theta X_{i}}\right] \\
& =e^{-\theta T} \prod_{i=1}^{k}\left(p_{i} e^{\theta}+1-p_{i}\right) \\
& \leq e^{-\theta T}\left(\frac{1}{k} \sum_{i=1}^{k}\left(p_{i} e^{\theta}+1-p_{i}\right)\right)^{k} \\
& \leq e^{-\theta T}\left(\frac{p}{k}\left(e^{\theta}-1\right)+1\right)^{k},
\end{aligned}
$$


where the second inequality follows from the fact that the geometric mean of nonnegative numbers is at most their arithmetic mean and the last inequality follows for $\theta>0$, which is true for the choice of $\theta=\log ((k-p) T /(p(k-T)))$ for any $T>p$. Then, we get

$$
\begin{aligned}
\mathbb{P}\left[\sum_{i=1}^{k} X_{i} \geq T\right] & \leq\left(\frac{p(k-T)}{(k-p) T}\right)^{T}\left(\frac{(k-p) T}{(k-T) k}+1-\frac{p}{k}\right)^{k} \\
& =\left(\frac{p}{T}\right)^{T}\left(\frac{k-p}{k-T}\right)^{k-T} \\
& =\left(\frac{p}{T}\right)^{T}\left(1+\frac{T-p}{k-T}\right)^{k-T} \\
& \leq\left(\frac{p}{T}\right)^{T} e^{T-p} \\
& =\exp \left(-T \log \left(\frac{T}{p}\right)+T-p\right) .
\end{aligned}
$$

Proof of Theorem 3.1. The proof of the theorem consists of two parts, which give upper bounds for probability of Type I and Type II errors. For any user $u$ and round $l$, let $\Xi_{u}(l)$ be the Bernoulli random variable with mean $\xi_{u}$ indicating if the feedback given by user $u$ in round $l$ is in error.

Type I error. The algorithm makes a Type I error if it declares an objective recommendation engine to be biased. This section shows that the probability that the algorithm makes a Type I error is low.

Suppose that the recommendation engine uses an objective recommendation algorithm. We first bound the probability that BiAD accepts $H_{1}$ in round $t$. Recall that the algorithm accepts $H_{1}$ in round $t$ if $S(t) \geq T(t)$ and that

$$
S(t)=\max _{\{\hat{\mathcal{A}} \subseteq[m]:|\hat{\mathcal{A}}|=\hat{A}(t)\}} \sum_{i \in \hat{\mathcal{A}}} B_{i}(t) .
$$

Consider a fixed $\hat{\mathcal{A}} \subseteq[m]$ such that $|\hat{\mathcal{A}}|=\hat{A}(t)$. We first bound the probability that $\sum_{i \in \hat{\mathcal{A}}} B_{i}(t) \geq T(t)$ and then use a union bound over all possible $\hat{\mathcal{A}}$ to obtain an upper bound on the probability that $S(t) \geq T(t)$. Recall that $\Xi_{u}(l)$ is equal to 1 if the feedback given by user $u$ in round $l$ is in error and 0 otherwise. Let us define

$$
X_{u}(l):=\sum_{i \in \hat{\mathcal{A}}} \mathbb{1}_{u i}(l)\left(\mathbb{1}\left\{R_{u i} \geq \eta\right\} \Xi_{u}(l)+\mathbb{1}\left\{R_{u i}<\eta\right\}\left(1-\Xi_{u}(l)\right)\right) .
$$

Note that $X_{u}(l)$ is equal to 1 if, in round $l$, an item from set $\hat{\mathcal{A}}$ is recommended to player $u$ and the player declares it ineffective. It is equal to 0 otherwise. Given $\{\mathbf{W}(l), \hat{\mathbf{R}}(l), l \in$ $[t]\}$, we have that $\left\{X_{u}(l), l \in[t], u \in[n]\right\}$ are Bernoulli random variables independent of each other. For every $1 \leq u \leq n$ and $1 \leq l \leq t$, the mean of $X_{u}(l)$ can be bounded as follows:

$$
\begin{aligned}
\mathbb{E}\left[X_{u}(l) \mid\left\{\mathbf{W}\left(l^{\prime}\right), \hat{\mathbf{R}}\left(l^{\prime}\right)\right\}_{l^{\prime}=1}^{t}\right] & \leq \sum_{i \in \hat{\mathcal{A}}} W_{u i}(l)\left(\xi_{u}+\mathbb{1}\left\{R_{u i}<\eta\right\}\right)\left(1-\xi_{u}\right) \\
& \leq \xi_{u}+P_{u}^{\hat{\mathcal{A}}}(l)\left(1-\xi_{u}\right)
\end{aligned}
$$


where the inequality follows from the upper bound for $W_{u i}(l)$ from Lemma 6.1 and the definition of $P_{u}^{\hat{\mathcal{A}}}(l)((7))$. From (6) and Condition (I)a, we have

$$
\sum_{u=1}^{n} \sum_{l=1}^{t} \xi_{u}+\mathbb{E}\left[P_{u}^{\hat{\mathcal{A}}}(l)\right]\left(1-\xi_{u}\right) \leq p(t) .
$$

Note that since the noise in the rating estimates are independent across time and users, $\left\{P_{u}^{\hat{\mathcal{A}}}(l), l \in[t], u \in[n]\right\}$ are independent random variables. Therefore, we can use the Chernoff bound in Lemma 6.2 to obtain a probabilistic upper bound on their sum. By Condition $(\mathrm{I}) \mathrm{b}, p(t)<n t$. In addition, to prove that $\hat{p}(t)<n t$, we consider the following two cases:

(i): $\beta(t) \geq 1$

$$
\begin{aligned}
\hat{p}(t) & =\exp \left(1+W\left(\frac{\beta(t)}{e}\right)\right) p(t) \\
& =\frac{\beta(t) p(t)}{W\left(\frac{\beta(t)}{e}\right)} \\
& \leq \frac{(\hat{A}(t)+c) \log m}{\left.W \frac{\beta(t)}{e}\right)} \\
& \leq 6 \hat{A}(t) \log m \\
& <n t
\end{aligned}
$$

for $n$ large enough by Condition (I)c. The second inequality follows from the fact that $\beta(t) \geq 1$ implies that $\left(6 W\left(\frac{\beta(t)}{e}\right)-1\right) \hat{A}(t) \geq 0.5=c$.

(ii): $\beta(t)<1$

$$
\begin{aligned}
\hat{p}(t) & =\exp \left(1+W\left(\frac{\beta(t)}{e}\right)\right) p(t) \\
& <4 p(t) \\
& \leq n t,
\end{aligned}
$$

where, the first inequality follows from the fact that $\beta(t)<1$ implies that $\exp (1+$ $\left.W\left(\frac{\beta(t)}{e}\right)\right)<4$ and the second inequality follows from Condition (I)b.

Applying Lemma 6.2 gives

$$
\mathbb{P}\left[\sum_{u=1}^{n} \sum_{l=1}^{t} \xi_{u}+P_{u}^{\hat{\mathcal{A}}}(l)\left(1-\xi_{u}\right) \geq \hat{p}(t)\right] \leq \exp \left(-\hat{p}(t)\left(\log \left(\frac{\hat{p}(t)}{p(t)}\right)-1\right)-p(t)\right) .
$$

Now, by the definition of Lambert-W function,

$$
\hat{p}(t)\left(\log \left(\frac{\hat{p}(t)}{p(t)}\right)-1\right) \geq(\hat{A}(t)+c) \log m-p(t),
$$

which further implies that

$$
\mathbb{P}\left[\sum_{u=1}^{n} \sum_{l=1}^{t} \xi_{u}+P_{u}^{\hat{A}}(l)\left(1-\xi_{u}\right) \geq \hat{p}(t)\right] \leq \exp (-(\hat{A}(t)+c) \log m) .
$$


We now proceed to obtain a probabilistic upper bound for the sum $\sum_{u=1}^{n} \sum_{l=1}^{t} X_{u}(l)$. By inequality (12), the sum of the corresponding means has the following upper bound:

$$
\sum_{u=1}^{n} \sum_{l=1}^{t} \mathbb{E}\left[X_{u}(l) \mid\left\{\mathbf{W}\left(l^{\prime}\right), \hat{\mathbf{R}}\left(l^{\prime}\right)\right\}_{l^{\prime}=1}^{t}\right] \leq \sum_{u=1}^{n} \sum_{l=1}^{t} \xi_{u}+P_{u}^{\hat{\mathcal{A}}}(l)\left(1-\xi_{u}\right) .
$$

Since $\left\{X_{u}(l), l \in[t], u \in[n]\right\}$ are independent Bernoulli random variables given $\{\mathbf{W}(l), \hat{\mathbf{R}}(l), l \in[t]\}$, Lemma 6.2 can be used as before. If $\sum_{u=1}^{n} \sum_{l=1}^{t} \xi_{u}+P_{u}^{\hat{\mathcal{A}}}(l)\left(1-\xi_{u}\right) \leq$ $\hat{p}(t)$, then Lemma 6.2 gives

$$
\mathbb{P}\left[\sum_{u=1}^{n} \sum_{l=1}^{t} X_{u}(l) \geq T(t) \mid\left\{\mathbf{W}\left(l^{\prime}\right), \hat{\mathbf{R}}\left(l^{\prime}\right)\right\}_{l^{\prime}=1}^{t}\right] \leq \exp (-(\hat{A}(t)+c) \log m)
$$

The preceding upper bound can be derived in exactly the same manner as the upper bound in (13). Combining this upper bound with inequality (13), we have

$$
\begin{aligned}
\mathbb{P}\left[\sum_{u=1}^{n} \sum_{l=1}^{t} X_{u}(l) \geq T(t)\right] \leq & \mathbb{P}\left[\sum_{u=1}^{n} \sum_{l=1}^{t} X_{u}(l) \geq T(t) \mid \sum_{u=1}^{n} \sum_{l=1}^{t} \xi_{u}+P_{u}^{\hat{\mathcal{A}}}(l)\left(1-\xi_{u}\right) \leq \hat{p}(t)\right] \\
& +\mathbb{P}\left[\sum_{u=1}^{n} \sum_{l=1}^{t} \xi_{u}+P_{u}^{\hat{\mathcal{A}}}(l)\left(1-\xi_{u}\right) \geq \hat{p}(t)\right] \\
\leq & 2 \exp (-(\hat{A}(t)+c) \log m) .
\end{aligned}
$$

Now recall that $B_{i}(t)$ is the number of players who have rated item $i$ ineffective up to round $t$, which can be mathematically written as $B_{i}(t)=\sum_{u=1}^{n} \sum_{l=1}^{t} \mathbb{1}_{u i}(l) \cdot \mathbb{1}\left\{R_{u i}<\eta\right\}$. Using definition (11), we have

$$
\sum_{i \in \hat{\mathcal{A}}} B_{i}(t)=\sum_{u=1}^{n} \sum_{l=1}^{t} X_{u}(l)
$$

which gives us the following equivalent form of inequality (15):

$$
\mathbb{P}\left[\sum_{i \in \hat{\mathcal{A}}} B_{i}(t) \geq T(t)\right] \leq 2 \exp (-(\hat{A}(t)+c) \log m)
$$

We can now take a union bound over all possible $\hat{\mathcal{A}}$ to bound the probability that BiAD accepts $H_{1}$ in round $t$ : 


$$
\begin{aligned}
\mathbb{P}[S(t) \geq T(t)] & =\mathbb{P}\left[\bigcup_{\{\hat{\mathcal{A}} \subseteq[m]:|\hat{\mathcal{A}}|=\hat{A}(t)\}}\left\{\sum_{i \in \hat{\mathcal{A}}} B_{i}(t) \geq T(t)\right\}\right] \\
& \leq \sum_{\{\hat{\mathcal{A}} \subseteq[m]:|\hat{\mathcal{A}}|=\hat{A}(t)\}} \mathbb{P}\left[\sum_{i \in \hat{\mathcal{A}}} B_{i}(t) \geq T(t)\right] \\
& \leq 2\left(\begin{array}{c}
m \\
\hat{A}(t)
\end{array}\right) \exp (-(\hat{A}(t)+c) \log m) \\
& \leq 2 \exp (-c \log m) \\
& =2 m^{-c},
\end{aligned}
$$

where the second inequality follows from (16). Further taking a union bound over all rounds,

$$
\begin{aligned}
\mathbb{P}[\text { Type I Error }] & =\mathbb{P}\left[\cup_{t=1}^{Q(m)} S(t) \geq T(t)\right] \\
& \leq \sum_{t=1}^{Q(m)} \mathbb{P}[S(t) \geq T(t)] \\
& \leq 2 Q(m) m^{-c} .
\end{aligned}
$$

This shows that BiAD declares an objective recommendation engine as biased with probability $O\left(\frac{Q(m)}{\sqrt{m}}\right)$ for the choice of $c=1 / 2$.

Type II error. The algorithm makes a Type II error if it does not detect a biased recommendation engine-that is, it declares $H_{0}$ when $H_{1}$ is true. Suppose that the recommendation engine is biased with an ad pool $\mathcal{A}$ of size $|\mathcal{A}|=A$. By Condition (II)a, $\mathrm{BiAD}$ has at least $A$ rounds of feedback. We prove that the total number of ad recommendations declared ineffective by the $n$ players until round $A$ is at least $\gamma \Omega(n A)$ with high probability. Let $\delta \in(0,1)$ be the constant given by Condition (II)b. For any $1 \leq u \leq n$, let $Y_{u}(l)=1$ if the biased recommendation engine decides to recommend from the ad pool to user $u$ in round $l$. Since $\left\{\left(1-\Xi_{u}(l)\right) Y_{u}(l), l \in[A], u \in[n]\right\}$ are independent Bernoulli random variables with mean $\left\{\left(1-\xi_{u}\right) \gamma, u \in[n]\right\}$, we have

$$
\mathbb{P}\left[\sum_{u=1}^{n} \sum_{l=1}^{A}\left(1-\Xi_{u}(l)\right) Y_{u}(l)<(1-\delta / 2)\left(1-c^{\prime}\right) \gamma A n\right] \leq e^{-\frac{\delta^{2}}{8}\left(1-c^{\prime}\right) \gamma A n},
$$

where the inequality follows from a version of the Chernoff bound given in Mitzenmacher and Upfal [2005] for sum of independent and identically distributed Bernoulli random variables using Condition (I)a. Note that $\sum_{u=1}^{n} \sum_{l=1}^{A}\left(1-\Xi_{u}(l)\right) \sum_{i \in \mathcal{A}} \mathbb{1}_{u i}(l)$ is the total number of recommendations from the ad pool $\mathcal{A}$ that were rated without error by the players and also that $\sum_{i \in \mathcal{A}} \mathbb{1}_{u i}(l) \geq Y_{u}(l)$. Therefore,

$$
\mathbb{P}\left[\sum_{u=1}^{n} \sum_{l=1}^{A}\left(1-\Xi_{u}(l)\right) \sum_{i \in \mathcal{A}} \mathbb{1}_{u i}(l)<(1-\delta / 2)\left(1-c^{\prime}\right) \gamma n A\right] \leq e^{-\frac{\delta^{2}}{8}\left(1-c^{\prime}\right) \gamma n A} .
$$

The detection algorithm makes the correct decision if in round $t, S(t) \geq T(t)$ for some $t \leq Q(m)$. We show that $S(A) \geq T(A)$ with high probability. Since $A \leq Q(m)$, the algorithm makes the correct decision with high probability. Now suppose that the total number of ad recommendations rated without error by the players until round $A$ is at 
least $(1-\delta / 2)\left(1-c^{\prime}\right) \gamma n A$. Since by Condition (II)c the total number of effective ads to the $n$ players is $o(\gamma n A)$, the total number of recommendations from the ad pool rated ineffective until round $A$ is $\gamma \Omega(n A)$. Consequently,

$$
\begin{aligned}
S(A) & =\max _{\{\hat{\mathcal{A}} \subseteq[m]:|\hat{\mathcal{A}}|=A\}} \sum_{i \in \hat{\mathcal{A}}} B_{i}(t) \\
& \geq \sum_{i \in \mathcal{A}} B_{i}(t) \\
& \geq(1-\delta / 2)\left(1-c^{\prime}\right) \gamma n A-o(\gamma n A) \\
& \geq(1-\delta)\left(1-c^{\prime}\right) \gamma n A,
\end{aligned}
$$

for $n$ large enough. To prove that $T(A)$ does not exceed the right-hand side of inequality (18), we consider the following cases:

(i) $\hat{\beta}(A) \geq e$

Since $W(\cdot)$ is an increasing function in $[0, \infty)$, we have $W\left(\frac{\hat{\beta}(A)}{e}\right) \geq W(1)>\frac{1}{2}$. Now,

$$
\begin{aligned}
T(A) & =\exp \left(1+W\left(\frac{\hat{\beta}(A)}{e}\right)\right) \hat{p}(A) \\
& =\frac{\hat{\beta}(A)}{W\left(\frac{\hat{\beta}(A)}{e}\right)} \hat{p}(A) \\
& \leq \frac{(A+c) \log m}{W(1)} \\
& \left.=\frac{(A+c) \log m}{W(1) A} \frac{\ln (n A)}{n}\right)^{2} \frac{\log m}{n}(n A),
\end{aligned}
$$

where the second equality follows from the definition of the Lambert $\mathrm{W}$ function, and the first inequality follows by using the definition of $\hat{\beta}(A)$ given by (3).

(ii) $\hat{\beta}(A)<e, \beta(A) \geq e$

$$
\begin{aligned}
\hat{p}(A) & =\exp \left(1+W\left(\frac{\beta(A)}{e}\right)\right) p(A) \\
& =\frac{\beta(A)}{W\left(\frac{\beta(A)}{e}\right)} p(A) \\
& \leq \frac{(A+c) \log m}{W(1)} \\
T(A) & =\exp \left(1+W\left(\frac{\hat{\beta}(A)}{e}\right)\right) \hat{p}(A) \\
& \leq \exp (1+W(1)) \hat{p}(A) \\
& =\frac{\exp (1+W(1))}{W(1)} \frac{(A+c)}{A} \frac{\log m}{n}(n A) \\
& \leq\left(\frac{e}{W(1)}\right)^{2} \frac{\log m}{n}(n A) .
\end{aligned}
$$


(iii) $\hat{\beta}(A)<e, \beta(A)<e$

$$
\begin{aligned}
T(A) & =\exp \left(1+W\left(\frac{\hat{\beta}(A)}{e}\right)\right) \hat{p}(A) \\
& \leq \exp (1+W(1)) \hat{p}(A) \\
& \leq \exp (2+2 W(1)) p(A) \\
& =\left(\frac{e}{W(1)}\right)^{2} \frac{p(A)}{n A} n A .
\end{aligned}
$$

Combining the results from the preceding three cases with inequality (18) and Condition (II)b gives that $S(A) \geq T(A)$ for $n$ large enough. Therefore, the algorithm declares the correct hypothesis in round $A$ if the total number of ad recommendations rated without error by the players until round $A$ is at least $(1-\delta / 2)\left(1-c^{\prime}\right) \gamma n A$. We can therefore use the concentration inequality in (17) to bound the probability of Type II error

$$
\begin{aligned}
\mathbb{P}[\text { Type II Error] } & \leq \mathbb{P}[S(A)<T(A)] \\
& \leq \mathbb{P}\left[\sum_{u=1}^{n} \sum_{l=1}^{A}\left(1-\Xi_{u}(l)\right) \sum_{i \in \mathcal{A}} \mathbb{1}_{u i}(l)<(1-\delta / 2)\left(1-c^{\prime}\right) \gamma n A\right] \\
& \leq e^{-\frac{\delta^{2}}{8}\left(1-c^{\prime}\right) \gamma n A} \\
& =e^{-\Omega\left(\left(1-c^{\prime}\right) \gamma n\right)} .
\end{aligned}
$$

This shows that the probability of Type II error decays exponentially with the number of players and the bias probability.

\section{CONCLUSION}

We propose an algorithm that can identify a biased recommendation engine that systematically favors a few sponsored advertisements over other genuine recommendations. We formulate a probabilistic model for recommender systems and give theoretical guarantees for our detection algorithm based on this model. Specifically, we show that the probability of missed detection and false positives are low for recommender systems with large databases. We show through simulations that the algorithm performs well for many datasets and different types of recommendation algorithms. In an age when both personalization and advertising have become quite prevalent, this kind of anomaly detection algorithm is relevant in a wide variety of scenarios. We demonstrate how our detection algorithm can be applied to problems such as identification of search engine bias and pharmaceutical lobbying. It would be interesting to investigate ways of deploying such an anomaly detection mechanism in practical settings.

\section{REFERENCES}

American Association of Advertising Agencies. 2009. Self-Regulatory Principles for Online Behavioral Advertising. Retrieved October 15, 2016, from http://www.aboutads.info/resource/download/sevenprinciples-07-01-09.pdf.

John Abraham. 2002. The pharmaceutical industry as a political player. Lancet 360, 9344, 1498-1502.

Deepak Agarwal. 2007. Detecting anomalies in cross-classified streams: A Bayesian approach. Knowledge and Information Systems 11, 1, 29-44.

Charu C. Aggarwal and Philip S. Yu. 2001. Outlier detection for high dimensional data. ACM SIGMOD Record 30, 2, 37-46.

Reed Albergotti. 2014. Facebook to clean up news feeds. Wall Street Journal. Retrieved October 15, 2016, from http://www.wsj.com/articles/facebook-to-clean-up-news-feeds-1416002403. 
Daniel Barbara, Ningning Wu, and Sushil Jajodia. 2001. Detecting novel network intrusions using Bayes estimators. In Proceedings of the 1st SIAM International Conference on Data Mining (SDM'01). 1-17.

Joeran Beel, Stefan Langer, and Marcel Genzmehr. 2013. Sponsored vs. organic (research paper) recommendations and the impact of labeling. In Research and Advanced Technology for Digital Libraries. Springer, 391-395.

James Bennett and Stan Lanning. 2007. The Netflix prize. In Proceedings of the KDD Cup and Workshop, Vol. 2007. 35.

Dirk Bergemann and Alessandro Bonatti. 2011. Targeting in advertising markets: Implications for offline versus online media. RAND Journal of Economics 42, 3, 417-443.

David Blumenthal. 2004. Doctors and drug companies. New England Journal of Medicine 351, 18, 1885-1890.

Richard J. Bolton and David J. Hand. 2002. Statistical fraud detection: A review. Statistical Science 17, 3, 235-249.

Robin Burke, Bamshad Mobasher, Chad Williams, and Runa Bhaumik. 2006. Classification features for attack detection in collaborative recommender systems. In Proceedings of the 12th ACM SIGKDD International Conference on Knowledge Discovery and Data Mining. ACM, New York, NY, 542-547.

Varun Chandola, Arindam Banerjee, and Vipin Kumar. 2009. Anomaly detection: A survey. ACM Computing Surveys 41, 3, 15.

Aidan Crook and Sanaz Ahari. 2011. Making search yours. Bing Blogs. Retrieved October 15, 2016, from http://blogs.bing.com/search/2011/02/10/making-search-yours.

Zhicheng Dou, Ruihua Song, and Ji-Rong Wen. 2007. A large-scale evaluation and analysis of personalized search strategies. In Proceedings of the 16th International Conference on World Wide Web. ACM, New York, NY, 581-590.

Lancet Editorial. 2005. The UK drug industry: Responsible, ethical, and professional? Lancet 366, 9500, 1828.

Dag Elgesem. 2008. Search engines and the public use of reason. Ethics and Information Technology 10, 4, $233-242$.

eMarketer. 2014. Native Advertising Roundup. Retrieved October 15, 2016, from https://www.emarketer.com/ public_media/docs/eMarketer_Native_Advertising_Roundup.pdf.

Robert M. Entman. 2007. Framing bias: Media in the distribution of power. Journal of Communication 57, $1,163-173$.

Robert Epstein. 2014. How Google could end democracy. U.S. News and World Report. Retrieved October 15, 2016, from http://www.usnews.com/opinion/articles/2014/06/09/how-googles-search-rankings-couldmanipulate-elections-and-end-democracy.

Federal Trade Commission. 1983. FTC Policy Statement on Deception. Retrieved October 15, 2016, from https://www.ftc.gov/public-statements/1983/10/ftc-policy-statement-deception.

Federal Trade Commission. 2013. .com Disclosures: How to Make Effective Disclosures in Digital Advertising. Retrieved October 15, 2016, from https://www.ftc.gov/system/files/documents/plain-language/ bus41-dot-com-disclosures-information-about-online-advertising.pdf.

Adriane Fugh-Berman and Shahram Ahari. 2007. Following the script: How drug reps make friends and influence doctors. PLoS Medicine 4, 4, e150.

Pedro Galeano, Daniel Peña, and Ruey S. Tsay. 2006. Outlier detection in multivariate time series by projection pursuit. Journal of the American Statistical Association 101, 474, 654-669.

Anindya Ghose and Sha Yang. 2009. An empirical analysis of search engine advertising: Sponsored search in electronic markets. Management Science 55, 10, 1605-1622.

Ben Goldacre. 2014. Bad Pharma: How Drug Companies Mislead Doctors and Harm Patients. Macmillan.

Laura A. Granka. 2010. The politics of search: A decade retrospective. Information Society 26, 5, 364-374.

Deepali Gupta. 2015. CCI charges Google with rigging search results; Flipkart, Facebook corroborate complaints. Economic Times. Available at http://economictimes.indiatimes.com.

Simon Hawkins, Hongxing He, Graham Williams, and Rohan Baxter. 2002. Outlier detection using replicator neural networks. In Data Warehousing and Knowledge Discovery. Springer, 170-180.

Zengyou He, Xiaofei Xu, and Shengchun Deng. 2003. Discovering cluster-based local outliers. Pattern Recognition Letters 24, 9, 1641-1650.

Hexagram. 2014. State of Native Advertising 2014. Retrieved October 15, 2016, from http:// stateofnativeadvertising.hexagram.com/.

Bryan Horling and Matthew Kulick. 2009. Personalized search for everyone. Google Official Blog. Retrieved October 15, 2016, from https://googleblog.blogspot.com/2009/12/personalized-search-for-everyone.html. 
Zan Huang, Daniel D. Zeng, and Hsinchun Chen. 2007. Analyzing consumer-product graphs: Empirical findings and applications in recommender systems. Management Science 53, 7, 1146-1164.

Interactive Advertising Bureau. 2010. Major Marketing/Media Trade Groups Launch Program to Give Consumers Enhanced Control Over Collection and Use of Web Viewing Data for Online Behavioral Advertising. Available at https://www.iab.com.

Interactive Advertising Bureau. 2013. IAB. The Native Advertising Playbook. Retrieved October 15, 2016, from http://www.iab.net/media/file/IAB-Native-Advertising-Playbook2.pdf.

Lucas D. Introna and Helen Nissenbaum. 2000. Shaping the Web: Why the politics of search engines matters. Information Society 16, 3, 169-185.

R. H. Keshavan, A. Montanari, and S. Oh. 2010. Matrix completion from a few entries. IEEE Transactions on Information Theory 56, 6, 2980-2998.

Subhashini Krishnasamy, Rajat Sen, Sewoong Oh, and Sanjay Shakkottai. 2015. Detecting sponsored recommendations. In Proceedings of the 2015 ACM International Conference on Measurement and Modeling of Computer Systems (SIGMETRICS'15). ACM, New York, NY.

Christopher Kruegel and Giovanni Vigna. 2003. Anomaly detection of Web-based attacks. In Proceedings of the 10th ACM Conference on Computer and Communications Security. ACM, New York, NY, 251-261.

Khaled Labib and Rao Vemuri. 2002. NSOM: A Real-Time Network-Based Intrusion Detection System Using Self-Organizing Maps. Technical Report. Department of Applied Science, University of California, Davis.

Sébastien Lahaie, David M. Pennock, Amin Saberi, and Rakesh V. Vohra. 2007. Sponsored search auctions. In Algorithmic Game Theory, N. Nisan, T. Roughgarden, E. Tardos, and V. Vazirani (Eds.). Stanford University, Stanford, CA, 699-716.

C. Seth Landefeld and Michael A. Steinman. 2009. The neurontin legacy-marketing through misinformation and manipulation. New England Journal of Medicine 360, 2, 103.

Steven H. Landers and Ashwini R. Sehgal. 2004. Health care lobbying in the United States. American Journal of Medicine 116, 7, 474-477.

Aleksandar Lazarevic, Levent Ertöz, Vipin Kumar, Aysel Ozgur, and Jaideep Srivastava. 2003. A comparative study of anomaly detection schemes in network intrusion detection. In Proceedings of the 2003 SIAM International Conference on Data Mining (SDM'03). 25-36.

Lihong Li, Wei Chu, John Langford, and Robert E. Schapire. 2010. A contextual-bandit approach to personalized news article recommendation. In Proceedings of the 19th International Conference on World Wide Web. ACM, New York, NY, 661-670.

Zhouchen Lin, Minming Chen, and Yi Ma. 2010. The augmented lagrange multiplier method for exact recovery of corrupted low-rank matrices. arXiv:1009.5055.

Matthew V. Mahoney and Philip K. Chan. 2002. Learning nonstationary models of normal network traffic for detecting novel attacks. In Proceedings of the 8th ACM SIGKDD International Conference on Knowledge Discovery and Data Mining. ACM, New York, NY, 376-385.

Julian McAuley and Jure Leskovec. 2013. Hidden factors and hidden topics: Understanding rating dimensions with review text. In Proceedings of the 7th ACM Conference on Recommender Systems. ACM, New York, NY, 165-172.

Michael Mitzenmacher and Eli Upfal. 2005. Probability and Computing: Randomized Algorithms and Probabilistic Analysis. Cambridge University Press.

Bamshad Mobasher, Robin Burke, Runa Bhaumik, and Chad Williams. 2007. Toward trustworthy recommender systems: An analysis of attack models and algorithm robustness. ACM Transactions on Internet Technology 7, 4, 23.

Spiros Papadimitriou, Hiroyuki Kitagawa, Philip B. Gibbons, and Christos Faloutsos. 2003. Loci: Fast outlier detection using the local correlation integral. In Proceedings of the 19th International Conference on Data Engineering. IEEE, Los Alamitos, CA, 315-326.

Sridhar Ramaswamy, Rajeev Rastogi, and Kyuseok Shim. 2000. Efficient algorithms for mining outliers from large data sets. ACM SIGMOD Record 29, 427-438.

Paul Resnick, Neophytos Iacovou, Mitesh Suchak, Peter Bergstrom, and John Riedl. 1994. NetNews. In Proceedings of the 1994 ACM Conference on Computer Supported Cooperative Work. ACM, New York, NY, 175-186.

Paat Rusmevichientong and David P. Williamson. 2006. An adaptive algorithm for selecting profitable keywords for search-based advertising services. In Proceedings of the 7th ACM Conference on Electronic Commerce. ACM, New York, NY, 260-269.

Bernhard Schölkopf, John C. Platt, John Shawe-Taylor, Alex J. Smola, and Robert C. Williamson. 2001. Estimating the support of a high-dimensional distribution. Neural Computation 13, 7, 1443-1471. 
Anup Shah. 2010. Pharmaceutical corporations and medical research. Global Issues. Retrieved October 15, 2016, from http://globalissues.org/article/52/pharmaceutical-corporations-and-medical-research.

Qing Song, Wenjie Hu, and Wenfang Xie. 2002. Robust support vector machine with bullet hole image classification. IEEE Transactions on Systems, Man, and Cybernetics, Part C: Applications and Reviews $32,4,440-448$.

Herman Tavani. 2014. Search engines and ethics. In Stanford Encyclopedia of Philosophy. Retrieved October 15, 2016, from http://plato.stanford.edu/entries/ethics-search.

Catherine Tucker. 2012. Social advertising. Retrieved October 15, 2016, from https://papers.ssrn.com/ sol3/papers.cfm?abstract_id=1975897.

A. M. Turpin and J. B. Katz. 2008. System and method for implementing advertising in an online social network. US Patent App.12/011,880.

Sha Yang and Anindya Ghose. 2010. Analyzing the relationship between organic and sponsored search advertising: Positive, negative, or zero interdependence? Marketing Science 29, 4, 602-623.

Dit-Yan Yeung and Calvin Chow. 2002. Parzen-window network intrusion detectors. In Proceedings of the 16th International Conference on Pattern Recognition, Vol. 4. IEEE, Los Alamitos, CA, 385-388.

Dantong Yu, Gholamhosein Sheikholeslami, and Aidong Zhang. 2002. Findout: Finding outliers in very large datasets. Knowledge and Information Systems 4, 4, 387-412.

Michael Zimmer. 2006. The value implications of the practice of paid search. Bulletin of the American Society for Information Science and Technology 32, 2, 23-25.

Received April 2015; revised February 2016; accepted August 2016 